\title{
Sustainable Discoloration of Textile Chromo-Baths by Spent Mushroom Substrate from the Industrial Cultivation of Pleurotus ostreatus
}

\author{
Simona Di Gregorio ${ }^{1}$, Francesco Balestri ${ }^{1}$, Maria Basile ${ }^{2}$, Valentina Matteini ${ }^{2}$, Francesco Gini ${ }^{1}$, \\ Stefania Giansanti ${ }^{2}$, Maria Grazia Tozzi ${ }^{1}$, Riccardo Basosi ${ }^{2}$, Roberto Lorenzi ${ }^{1}$ \\ ${ }^{1}$ Department of Biology, University of Pisa, Pisa, Italy; ${ }^{2}$ Department of Chemistry, University of Siena, Siena, Italy. \\ Email: sdigregorio@biologia.unipi.it
}

Received January $24^{\text {th }}, 2010$; revised April 11 ${ }^{\text {th }}, 2010$; accepted April $12^{\text {th }}, 2010$.

\begin{abstract}
Synthetic dyes are recalcitrant to degradation and toxic to different organisms. Physical-chemical treatments of textile wastewaters are not sustainable in terms of costs. Biological treatments can be more convenient and the lignin-degrading extracellular enzymatic battery of basidiomycetes are capable to discolor synthetic dyes. Many basidiomycetes are edible mushrooms whose industrial production generates significant amount of spent mushroom substrate $(S M S)$ with residual high levels of lignin-degrading extracellular enzymatic activities. We have demonstrated that the low cost organic substrate, the SMS deriving from the cultivation of the basidiomycetes Pleurotus ostreatus, is able to discolor anthraquinonic, diazo and monoazo-dyes when incubated in dying chromo-reactive and chromo-acid baths containing surfactants and anti-foams, where the concentrations of the different dyes are exceeding the one recovered in the corresponding wastewaters. Laccase was the lignin-degrading extracellular enzyme involved in the discolouring process. The exploitation of the low cost SMS in the treatment of textile wastewaters is proposed. Accordingly, a toxicological assessment, based on a cyto-toxicity test on a human amnion epithelial cell line (WISH) and the estimation of the germination index (GI\%) of Lactuca sativa, Cucumis sativus and Sorghum bicolor, has been performed, showing the loss of toxicity of the chromo-baths after being discoloured by the SMS.
\end{abstract}

Keywords: Anthraquinonic Dye, Diazo Dye, Laccase, Monoazo Dye, White Rot Fungi, Spent Mushroom Substrate

\section{Introduction}

Synthetic dyes find application in different industrial divisions including textile industry. An annual consumption of about 0.7 million tons of synthetic dyes has been reported [1]. These compounds result to be recalcitrant to degradation and toxic for higher animals [2] and surface aquifers [3]. Textile industry alone accounts for two-thirds of the total dyestuff market. Accordingly, discoloration of textile wastewaters is one of the major environmental concerns since last decades. Physical-chemical treatments such as coagulation/adsorption, electrolysis or ozonation are sometime unsuccessful or very expensive and frequently producing large amounts of toxic wastes [4]. At the same time, the strong electron-withdrawing groups characterizing the chemical structure of dyes protect them by the attack of bacterial oxygenases [5], affecting the treatment of textile wastewaters by conventional activated sludge plants. On the other hand, anaerobic digestions of various dyes produce toxic amines [6], which make the processes not recommended, unless combined with subsequent aerobic treatments to oxidize the toxic intermediates [7].

In this scenario, the already reported capacities of fungi $[8,9]$ with respect to aerobic oxidation of synthetic azo-dyes [10,11] anthraquinones [12], and phthalocyanins [13], result to be of extreme interest. Ligninolytic basidiomycetes causing white rot on wood, were shown to be the most promising fungi because of their capacity to produce a complex array of lignin-degrading extra-cellular enzymes, with very low substrate specificity towards xenobiotics [9]. The ligninolytic battery of extracellular enzymes of the basidiomycetes $P$. ostreatus has been described as capable to transform a very broad spectrum of waste substrates [14-16] including textile dyes [17]. However, it is worth to mention that $P$. ostreatus and many other basidiomycetes are edible 
mushrooms and their industrial cultivation produces a significant amount of spent mushroom substrate (SMS), reported as harboring high levels of residual oxidative enzymatic activity [18]. Actually, an average of $5 \mathrm{~kg}$ of SMS, are produced for $1 \mathrm{~kg}$ of mushrooms on the market, and the P. ostreatus production has increased over $500 \%$ in the last ten years, ranking the second or the third position in the context of edible mushroom industrial production in the world [19]. Thus, SMS would be a low cost source of ligninolytic enzyme [20-22]. However, the costs of purification of the latter can still negatively influence the exploitation of the spent matrix in pollutants biodegradation. As far as we know, with the exception of the description of potential of the spent mushroom compost of Pleurotus pulmonarius in synthetic pentachlophenol contaminated waters [23], no report on the potential of the SMS from the cultivation of edible mushrooms as an oxidising matrix for the treatment of industrial effluents has been reported.

The aim of this work was the recover and direct exploitation of the low cost organic substrate, the SMS from the industrial production of $P$. ostreatus, to discolour complex chromo-baths used in textile industry. The feasibility of the process has been verified incubating the SMS directly in reactive and acid chromobaths, containing anthraquinonic, mono and diazo dyes, as single dyes or as a mix. In addition to dyes, the chromo-baths contained surfactants and anti-foams that are used as auxiliaries in the colouring process and normally released in textile wastewaters. Although usually not specifically investigated in terms of disappearance and generally used as biodegradable ingredients, surfactants and anti-foams might interfere with the discolouring process or contribute to the toxicity of the textile effluents. The progressive discolouration of the different complex chromo-baths by the SMS were recorded in parallel with the evaluation of the loss of the here recorded toxicity. This latter has been monitored by a cyto-test on a human amnion epithelial cell line (WISH) and the estimation of the germination index (GI\%) of Lactuca sativa, Cucumis sativus and Sorghum bicolor in presence of untreated and discoloured chromo-baths. The ligninolytic enzymes potentially involved in the discolouring and detoxifying process have been monitored.

\section{Materials and Methods}

\subsection{Chemicals, SMS, Seeds and Epithelial Cell Line}

Analytical grade chemicals were purchased from Sigma Aldrich (Milan, Italy). The chromo-reactive and chromoacid baths were provided by manufacturers, chemical components are reported in Table 1. The real structure of the different dyes and auxiliaries were not disclosed by the manufactures, that categorized the different chromobaths in relation to the general classification of antraquinonic, mono and diazo-group. Surfactants (Setavin group) and anti-foams (Kolassol group) were respectively classified as alkylamine ethoxylated and silicon based chemicals. The SMS of Pleurotus ostreatus was obtained by a local mushroom farm. The human amnion epithelial cell line (WISH) was kindly provided by Prof. Francesco Sgarrella, University of Sassari (Italy). Plant seeds were obtained from the USDA-ARS North Central Regional Plant Introduction Station, Iowa State University, Ames, IA, USA.

\subsection{Discoloration of the Chromo-Baths by the SMS}

The capacity of the SMS to discolor the chromo-baths was tested at $21 \pm 2^{\circ} \mathrm{C}$, in static and dynamic conditions (orbital shaking at $250 \mathrm{rpm}$ ) in sterile $2 \mathrm{~L}$ glass flasks incubated with plugs of SMS $(0.3 \mathrm{~cm}$ thick), visually homogenously colonized by the fungal mycelium. The

Table 1. Chemical composition of the chromo-baths

\begin{tabular}{|c|c|c|c|c|c|c|}
\hline Bath name & Dye name & $\mathrm{g} / 1$ & $\begin{array}{l}\text { Auxiliaries } \\
\text { Name }\end{array}$ & $\mathrm{g} / 1$ & $\begin{array}{c}\% \\
\text { vol/vol }\end{array}$ & $\lambda_{\max }$ \\
\hline \multirow{4}{*}{$\begin{array}{l}\text { Black Reactive } \\
\text { mix (BRM) }\end{array}$} & $\begin{array}{l}\text { Yellow } 39 \\
5-10 \% \mathrm{wt} / \mathrm{vol}\end{array}$ & 110 & $\begin{array}{l}\text { Kollasol } \\
\text { LO-BD }\end{array}$ & 4 & \multirow[t]{4}{*}{ 的 } & 590 \\
\hline & Red 136 & & Setavin RE & 20 & & \\
\hline & $\begin{array}{l}47-52 \% \mathrm{wt} / \mathrm{vol} \\
\text { (monoazo) }\end{array}$ & & $\begin{array}{l}80 \% \\
\text { Acetic acid }\end{array}$ & & & \\
\hline & $\begin{array}{l}\text { Black DM } 5594 \text { 43\% } \\
\text { wt/vol (disazo) }\end{array}$ & & & & & \\
\hline \multirow{3}{*}{$\begin{array}{l}\text { Blue Reactive } \\
\text { Bath } \\
\text { (BRaB) }\end{array}$} & \multirow{3}{*}{$\begin{array}{l}\text { Blue 3G } \\
\text { (anthraquinonic) }\end{array}$} & 11 & $\begin{array}{l}\text { Kollasol } \\
\text { LO-BD }\end{array}$ & 5 & \multirow{7}{*}{1.5} & 609 \\
\hline & & & Setavin RE & 20 & & \\
\hline & & & $80 \%$ & & & \\
\hline \multirow{2}{*}{$\begin{array}{l}\text { Blue Acid } \\
\text { bath } \\
\text { (BAaB) }\end{array}$} & \multirow{2}{*}{$\begin{array}{l}\text { Blue BLI } \\
\text { (anthraquinonic) }\end{array}$} & 11 & Setavin MSN & 7.5 & & 637 \\
\hline & & & $\mathrm{NH}_{4} \mathrm{SO}_{4}$ & 30 & & 595 \\
\hline \multirow{2}{*}{$\begin{array}{l}\text { Acid } \\
\text { red } \\
\text { (RAmaB) }\end{array}$} & \multirow{2}{*}{$\begin{array}{l}\text { Red FGN } \\
\text { (monoazo) }\end{array}$} & \multirow[t]{2}{*}{11} & Setavin MSN & 7.5 & & 505 \\
\hline & & & $\mathrm{NH}_{4} \mathrm{SO}_{4}$ & 30 & & \\
\hline
\end{tabular}


SMS was collected for a total amount of $130 \mathrm{~g}$ of fresh weight and submerged in the glass flask containing 500 $\mathrm{mL}$ of the coloring bath diluted in $150 \mathrm{mM} \mathrm{NaCl}$ solution in water. The Black chromo-Reactive Mix (BRM) was 10 and 5-fold diluted, the Blue chromo-Reactive anthraquinonic Bath (BRaB), the Blue chromo-Acid anthraquinonic Bath $(\mathrm{BAaB})$ and the Red chromo-Acid monoazo Bath (RAmaB) were 5-fold diluted. Two flasks (one set) were prepared for each dilution. One set of flasks was not inoculated with SMS, the other was inoculated with SMS previously autoclaved at $121^{\circ} \mathrm{C}, 1 \mathrm{~atm}$ for 20 minutes, respectively control sets for abiotic discoloration and for adsorption of the dye onto the SMS. A further set of flasks was prepared in sterile $150 \mathrm{mM} \mathrm{NaCl}$ solution in water to evaluate the contribution of the release of pigmentation from SMS to the increase in the spectral absorbance of flask supernatants in the visible range. At each time-point, three volumes of supernatant of each flask were collected and analyzed for UV-Vis absorption between 400-800 nm (Beckman Instruments, Fullerton, CA, USA). All the samples at time zero were diluted to give an absorbance $<1$ and the same dilution has been used for samples corresponding to the successive time points. The $150 \mathrm{mM} \mathrm{NaCl}$ solution served as blank. Percentage of discoloration with time of incubation was quantified as the decrease of areas under the absorbance spectra of flask supernatants at the different time-points of analysis and calculated against a baseline defined by the absorbance of supernatants from flasks without chromo-bath addition, at the corresponding time of analysis. The areas under the absorbance spectra were calculated by using PeakFit, Systat Software Inc., San Jose, California, USA. Decrease of the area under the spectra of supernatants deriving from flasks inoculated with autoclaved SMS with respect to the areas under spectra of supernatants from flask not inoculated with SMS was interpreted as percentage of adsorption of the dye onto SMS.

\subsection{Enzymatic Activity}

Ligninolytic oxidative capacity related to laccase (EC 1.10.3.2), manganese peroxidase (EC1.11.1.13), lignin peroxidase (EC1.11.1.14) and versatile peroxidase (EC1.11.1.16) activity were quantified, by a spectrophotometer based method, in sample volumes of each flask supernatant collected in triplicate. Enzymatic activities were calculated as specific activity. Total proteins were determined according to [24], using bovine serum albumin as standard. All enzyme assays were performed at 37 $\pm 0.5^{\circ} \mathrm{C}$. Linearity with time and protein concentration was observed for all enzyme activities assayed. Laccase activity was determined as described by [25]. Manganese peroxidase and versatile peroxidase activity were deter- mined as described by [26]. Lignin peroxidase activity was measured as described by [27].

Enzymatic activities were detected before chromo-bath amendments, at the moment of the amendment of chromo-baths and at the end of the discoloring process. Laccase stability at different $\mathrm{pH}$ of incubation was monitored on $20 \mu \mathrm{l}$ of control flask supernatant (no chromo-baths amended) incubated in different buffers and quantified using standard conditions at the time zero, after 3 and 24 hours of incubation. Laccase stability at different temperature was monitored on $50 \mu \mathrm{L}$ of control flask supernatant incubated for $15^{\prime}$ at different temperature. The effect of ionic strength on laccase activity was monitored in presence of increasing amounts of $\mathrm{NaCl}$ in the laccase mixture assay.

\subsection{Isolation of $\boldsymbol{P}$. ostreatus Mycelium}

The mycelium of $P$. ostreatus has been aseptically collected from the fungal fruiting body, streaked on a sterile potato dextrose agar plate and incubated for 1 week at $28^{\circ} \mathrm{C}$ in the dark. The mycelium was maintained, through periodic transfer at $4^{\circ} \mathrm{C}$ on potato dextrose agar plates in the presence of $0.5 \%$ yeast extract. Laccase activity has been monitored in liquid cultures prepared incubating 3 plugs $(1 \mathrm{~cm}$ diameter) of the aseptically collected mycelium, grown on the maintaining agar plates, in shaking flask $(125 \mathrm{rpm} / \mathrm{min})$ containing $250 \mathrm{ml}$ potato dextrose $(24 \mathrm{~g} / \mathrm{L})$ broth with $0.5 \%$ yeast extract at $28^{\circ} \mathrm{C}$ in the dark. At successive times, $1 \mathrm{~mL}$ of supernatant was collected in triplicate and analyzed for laccase activity.

\subsection{Native Protein Gel Electrophoresis in Gradient of Polyacrylamide}

A $5-30 \%$ polyacrylamide gradient gel was performed at an alkaline $\mathrm{pH}$ under non-denaturing conditions. The separating gel contained a gradient of acrylamide from 5 to $30 \%$, while the stacking gel contained $4 \%$ of acrylamide. The electrode reservoir solution was $25 \mathrm{mM}$ Tris-190 mM glycine (pH 8.4). A total of $60 \mathrm{ng}$ of proteins deriving from the supernatants of 1) P. ostreatus mycelium grown in potato dextrose broth; 2) SMS incubated in control flasks; 3) SMS incubated in 5 fold diluted blue chromo-reactive bath $(\mathrm{BRaB})$, were loaded on the gel that has been stained for laccase activity using 2,2'-azinobis-(3-ethylbenzthiazoline-6-sulfonic acid) (ABTS) as substrate. Sample supernatants were collected at the time of maximum laccase activity for 1).

\subsection{Seed Germination Test}

The germination indexes (GI\%) of Lactuca sativa, $\mathrm{Cu}$ cumis sativus, Sorghum bicolor seeds in presence of aliquots of undiluted different flask supernatants before and 
after the treatment with the $P$. ostreatus SMS were compared. Seed germination tests were performed in sterile Petri dish plates containing Whatman N.1 ashless filter imbibed with $10 \mathrm{ml}$ of 1) control solution, $150 \mathrm{mM} \mathrm{NaCl}$ in water; flask supernatants 2) before and 3) after the SMS incubation. The plates were kept for $120 \mathrm{hrs}$ in the dark at $25 \pm 1{ }^{\circ} \mathrm{C}$. The germination indexes were calculated from the number of germinated seeds and the corresponding root length values, according to the formula: $\mathrm{GI} \%=(\mathrm{Gs} \times \mathrm{Ls}) /(\mathrm{Gc} \times \mathrm{Lc}) \times 100$, where Gs was the mean number of germinated seeds and Ls the mean root length of germinated seeds in the sample; Gc was the mean number of germinated seeds and Lc the mean root length of the germinated seed in the control. The analyses were done in triplicate in plates containing 15 seeds for each set.

\subsection{Cytotoxic Test}

The established WISH cell line derived from human amnion epithelium has been routinely grown in RPMI medium with $10 \% \mathrm{FBS}$ and $2 \%$ antibiotics at $37 \pm 0.5^{\circ} \mathrm{C}$ in a humidified $5 \% \mathrm{CO}_{2} / 95 \%$ air atmosphere. The experiments were performed on $35 \mathrm{~mm}$ plates containing approximately 1.000 .000 of cells in RPMI complete medium maintained for 24 hours in the absence (control) or presence of volumes of the different flask supernatants corresponding to the dilutions of the chromo-baths used for the SMS discoloration. After incubation, plates were evaluated for the number of adhering cells to plates and for the trypan blue method [28] to evaluate the acute toxicity of the chromo-baths before and after discoloration with SMS.

\subsection{Statistical Treatment of Data}

The analysis of variance of the data (ANOVA), has been performed by using GraphPad InStat version 3.00 for Windows 95, GraphPad Software, San Diego California USA, to evaluate the effects of different parameters of incubation on the discolouring process. The means of the significantly different main effects were compared by the Duncan's test at the 5\% level using the Statistic program (Statsoft Inc., 1997).

\section{Results}

\subsection{Discoloration of the Chromo-Baths}

In order to evaluate the potential of $P$. ostreatus SMS to discolor the black chromo-reactive mix (BRM), two different dilutions of the latter were incubated in presence of the SMS. The incubation of the 10-fold diluted BRM was operated both in static and dynamic conditions. Discoloration of 10-fold diluted BRM, matched with a visible effect to the naked eye. The $97 \%$ of discoloration occurred in $24 \mathrm{hrs}$ (Figure 1(a)). The process has been observed only in presence of not autoclaved SMS and in dynamic incubation. In static condition a maximum of $8 \%$ of discoloration has been recorded.

Due to the high percentage of discoloration observed in dynamic incubation with the 10-fold dilution, a 5-fold dilution of BRM has been inoculated with the SMS only in dynamic incubation, because of the evidence that the oxygenation was mandatory for discoloration. After $24 \mathrm{hrs}$, the $65 \%$ of discoloration has been observed; the $80 \%$ was recorded after $110 \mathrm{hrs}$ of incubation (Figure 1(b)).
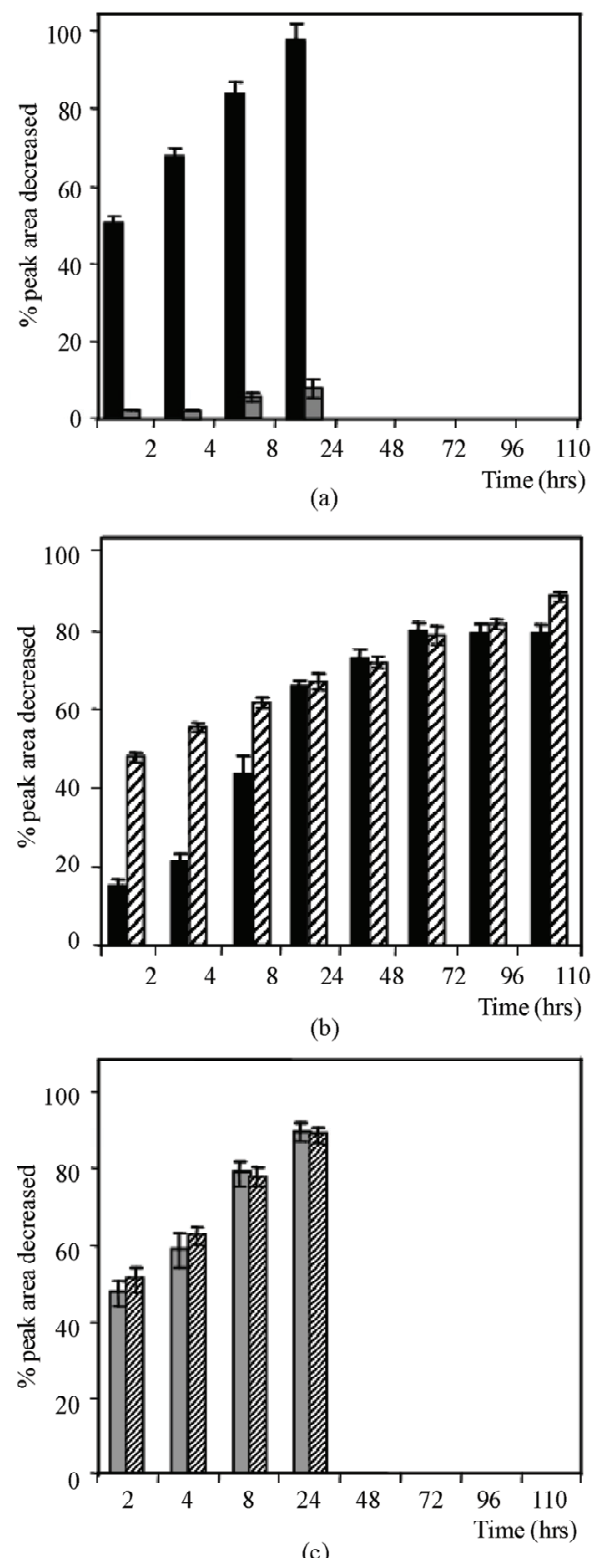

Figure 1. Panel (a) discoloration by the SMS of the 10-fold BRM, in static (grey bar) and dynamic incubation (black bar); Panel (b) discoloration of the 5-fold diluted BRM (black bar) and BRaB (dashed bar) in dynamic incubation; Panel (c) discoloration of 5-fold diluted BAaB, (grey bar) and RAmaB (dashed bar) in dynamic incubation 


\subsection{Enzymatic Activities and Native Gel Electrophoresis}

Enzymatic activities of lignin-degrading enzymes, measured before the addition of the chromo-baths to the discoloring sets of flasks, revealed that the lignin degrading battery of enzymes was limited to laccase. In fact no lignin peroxidase activity has been detected and the recorded laccase activity was predominant on the manganese and versatile peroxidase $(0.294 \pm 0.65 \mathrm{U} / \mathrm{mg}$ for laccase; $0.039 \pm 0.03 \mathrm{U} / \mathrm{mg}$ and $0.034 \pm 0.017 \mathrm{U} / \mathrm{mg}$ for manganese and versatile peroxidase respectively). The same enzymatic activity profile was recorded after the chromo-baths amendment and at the end of the discol- ouring process (Table 2). An increase of laccase specific activity either in presence or absence of chromo-baths has been recorded during the discolouring process (Table 2). No enzymatic activities have been detected in the supernatants of autoclaved SMS.

The laccase recovered from the supernatant of SMS incubated in the 5-fold diluted $\mathrm{BRaB}$ has been collected to be compared by native gel electrophoresis to 1) the laccase recovered from the set of flasks of control; 2) the supernatant of actively growing mycelium of $P$. osteauts in rich medium. Results obtained showed a very similar profile for the laccases recovered from the three supernatants (Figure 2(d)).

Table 2. Laccase activity recovered in the supernatants of the set of flasks of control $(C)$ and the different discolouring flasks

\begin{tabular}{|c|c|c|c|c|c|c|}
\hline $\begin{array}{c}\text { Laccase } \\
\text { activity U/mg }\end{array}$ & C flasks & $\begin{array}{c}\text { BRM } \\
\text { 10-fold diluted }\end{array}$ & $\begin{array}{c}\text { BRM } \\
\text { 5-fold diluted }\end{array}$ & $\begin{array}{c}\text { BRaB } \\
\text { 5-fold diluted }\end{array}$ & $\begin{array}{c}\text { BAaB } \\
\text { 5-fold diluted }\end{array}$ & $\begin{array}{c}\text { RAmaB } \\
\text { 5-fold diluted }\end{array}$ \\
\hline T0 & $0.267 \pm 0.042$ & $0.276 \pm 0.008$ & $0.363 \pm 0.040$ & $0.267 \pm 0.004$ & $0.248 \pm 0.007$ & $0.334 \pm 0.002$ \\
\hline TE & $0.396 * * * \pm 0.020$ & $0.328 * \pm 0.028$ & $0.581 * * * \pm 0.057$ & $0.346^{* *} \pm 0.007$ & $0.393 * * * \pm 0.005$ & $0.387 * \pm 0.010$ \\
\hline
\end{tabular}

T0, time of chromo-baths amendment; TE, end of the discoloring process. Statistical significance of difference recorded for laccase activity at the end vs. the beginning of the discoloring processes: $* * * \mathrm{p}<0.0001 ; * * \mathrm{p}<0.001 ; *_{\mathrm{p}}<0.05$. BRM, black reactive mix; BRaB, blu reactive bath; BAaB, blue acid bath, RAmaB red acid bath.

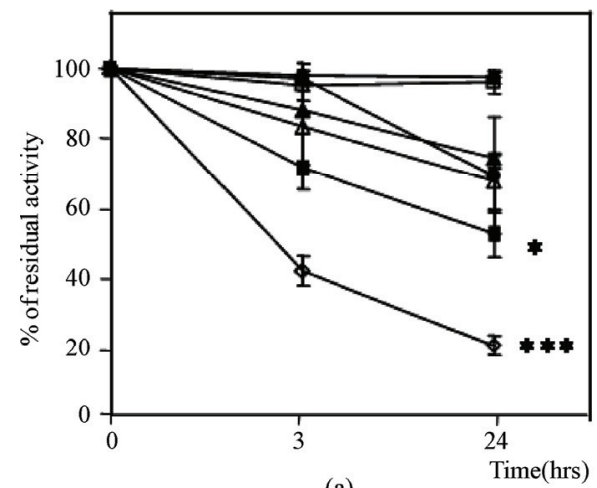

(a)

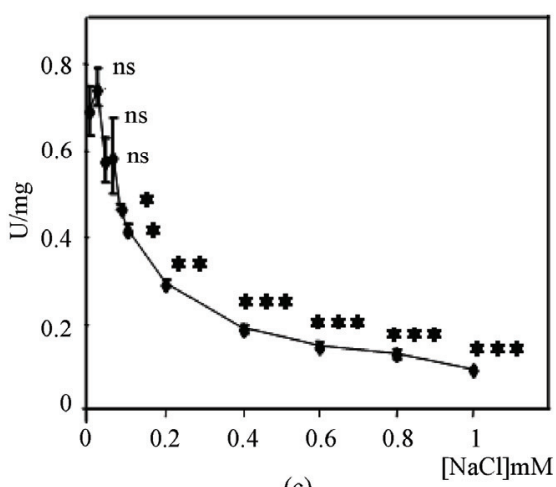

(c)

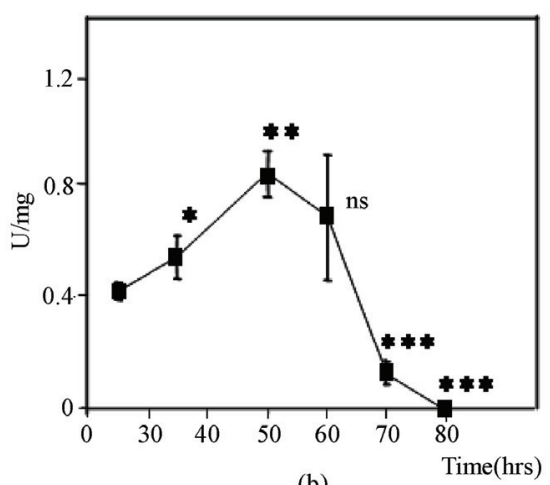

(b)

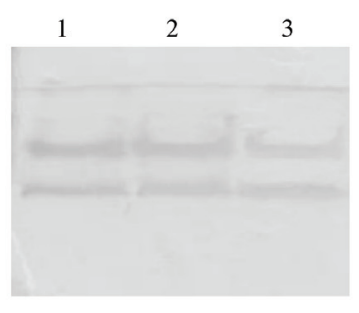

(d)

Figure 2. Residual laccase activity assayed in standard condition after exposure at different pH as function of time (panel a), after exposure for $30 \mathrm{~min}$ at different temperature (panel b) or assayed at different ionic strength (panel c). The laccase activity was measured on supernatant of SMS of set of flasks of control. Panel a: citrate buffer, pH $3(\diamond)$, acetate buffer, pH 4 ( $\square$ ), glycine buffer, pH $10(\Delta)$, tris buffer, pH $8(\bullet)$, acetate buffer, pH $5(\Delta)$, sodium phosphate buffer, pH $6(\square)$, sodium phosphate buffer, pH 7 ( $\bullet$. Statistical significance $* * * p<0.0001 ; * * p<0.001 ; * \mathbf{p}<0.05$. Panel d shows the zymogram of laccase isoenzymes derived from the supernatant of: lane 1, mycelium of $P$. ostreatus actively growing in potato dextrose broth; lane 2, SMS in control flasks, $150 \mathrm{mM} \mathrm{NaCl}$ in water, lane 3, SMS incubated in 5-fold diluted BRaB 
The stability of the laccase recovered from the set of flasks of control has been monitored with respect to $\mathrm{pH}$ and temperature, furthermore the effect exerted by ionic strength has been evaluated (Figures 2(a)-(c)). The enzyme, whose optimum $\mathrm{pH}$ was between 4 and 5 (results not shown), showed a significant stability in a range of pHs between 5 and 10 and after 30 min of incubation up to $60^{\circ} \mathrm{C}$. The laccase activity resulted to be $50 \%$ inhibited by $200 \mathrm{mM} \mathrm{NaCl}$.

\subsection{Germination and Cytotoxicity Test}

As a result of the germination tests, all the chromo-baths were extremely toxic because of the significant inhibition of seed germination that was nearly absent in the case of incubation in chromo-reactive baths for all the three plant species analyzed (Figures 3(a)-(d)). Acid baths were also extremely toxic $(\mathrm{GI} \%<50 \%)$, even though a certain percentage of germination has been observed (20\%) with the exception of Lactuca sativa that was strongly inhibited in germination by the Red chromo-acid bath (Figures 3(a)-(d)). The discoloration of all the chromo-baths determined the loss of toxicity of the discolored baths with a recover of the GI\% to values (nearly $100 \%$ ) communicated for the selected plant seeds by the USDA-ARS North Central Regional Plant Introduction Station (Figures 3(a)-(d)).

A human amnion epithelial cell line (WISH) has been used to determine the effect exerted by the chromo-baths on the cell viability. The WISH cells subjected to incubation with chromo-baths showed a significant decrease of the number of adhering (healthy) cells (Figure 3(e)) and a significant increase of the percentage of dead cells (stained by trypan blue) (Figure 3(f)) compared to control cells incubated in PBS 5-fold diluted in RPMI medium (control medium). The discoloration of the chromobaths by SMS incubation removed their toxic effect: the number of adhering cells and the percentage of dead cells, were not significantly different as compared to the control (Figures 3(e)-(f)).

\section{Discussion}

Both azo and anthraquinonic dyes are associated to a high ecotoxicity and high recalcitrance to discoloration $[12,29]$. Our results demonstrated that, in aerated (oxidative) conditions, a low cost organic substrate, the SMS from $P$. ostreatus cultivation, discolored industrial chromo-baths containing either monoazo, diazo and anthraquinonic dyes, either as single dyes or as a mix of them. Being interested in SMS exploitation for discolouring textile wastewaters, the capacity of the organic substrate has been tested on industrial chromo-baths, instead of aqueous solution of single synthetic dyes. In fact, the formers have a higher similarity to the real industrial wastewaters, since containing auxiliaries of the dying process (mainly surfactants, anti-foams and salts), that actually are also released in real wastewaters and potentially can interfere with the discolouring process. The dilution of the chromo-baths, here tested for discoloration, contained the different chemicals at concentrations definitely higher than the one recovered in the real wastewaters. In fact, they contained dyes and auxiliaries in concentration up to the $20 \%$ of the initial chromo-bath mass used in the dying process, while it is estimated that the amount of textile dyes released in wastewaters during the industrial process accounts for the $10 \%$ of the total used [30]. Thus, the SMS was capable to discolour different chromo-baths at similar or higher concentrations than the one recovered in real textile wastewaters. The efficiency of the SMS in the described process was different in relation to the different chromo-baths. Reactive baths resulted more recalcitrant than acid one, even though a comparison is either questionable because of the difference in chemical components of the different chromo-baths, and difficult since the real structures of the different dyes and chromo-baths auxiliaries, indicating their putative recalcitrance to biodegradation, were not provided by the manufacturers. However, in $24 \mathrm{hrs}$ the discoloration of every chromo-baths tested accounted for the $70-90 \%$ of the total. Abiotic discoloration and adsorption were excluded by the persistence of colours in sets of flasks not incubated with the SMS and in sets of flasks where every biological activity has been eliminated by autoclaving. Thus, the occurrence of a biotic process has been explored monitoring the ligninolytic activity of fungal extracellular enzymes, eventually associated to the grow of $P$. ostreatus on the SMS. Laccase resulted to be either the only enzymatic activity measurable from the incubation of the SMS in a saline solution and in presence of the different chromo-baths and the only enzymatic activity recovered during the time interval corresponding to the discoloration process. Actually during discoloration, laccase activity increased either in presence or absence of chromo-baths. Results obtained indicated the ligninolytic laccase as the enzymatic activity involved in the discolouring process. The hypothesis was confirmed by the nearly absence of discoloration in static condition, because in accordance with the known mechanism of catalytic reaction of the enzyme that uses molecular oxygen as electron donor to catalyze reactions consequently occurring in oxidative (aerated) conditions. The laccase recovered in the supernatant of discolouring baths showed peculiar traits. Laccases from basidiomycetes are generally considered acidic enzymes not active at basic $\mathrm{pH}$, characteristic actually not convenient for the exploitation of the enzyme in the treatment of textile wastewaters, which are essentially associated to neutral up to basic $\mathrm{pH}$. However, the laccase produced by $P$. ostreatus growing on the SMS, although displaying an acidic optimum of $\mathrm{pH}$, showed activity and high stability at neutral to strong basic $\mathrm{pH}$. A second 

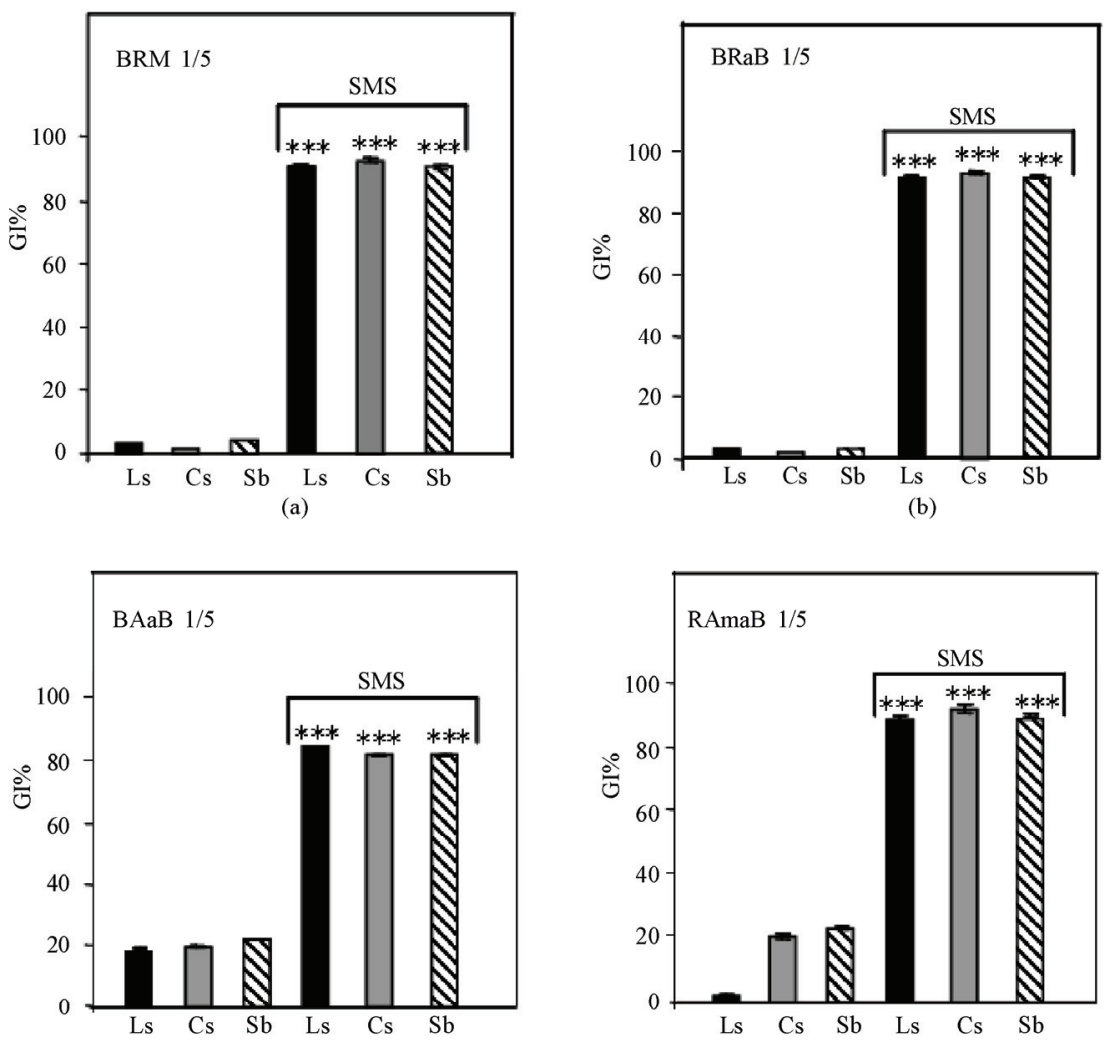

(c)

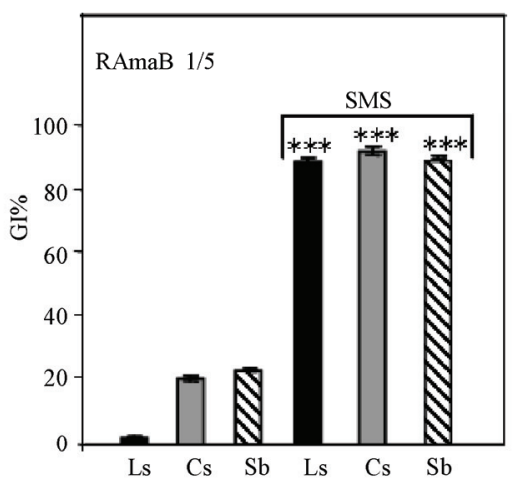

(d)

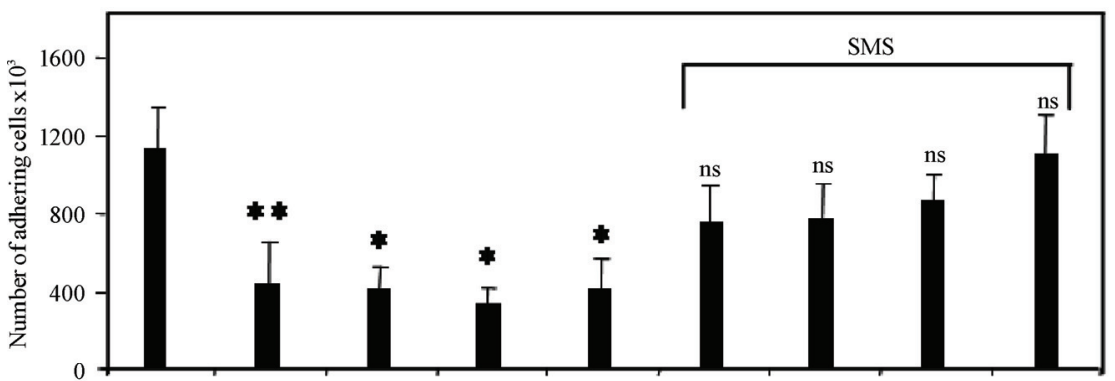

(e)

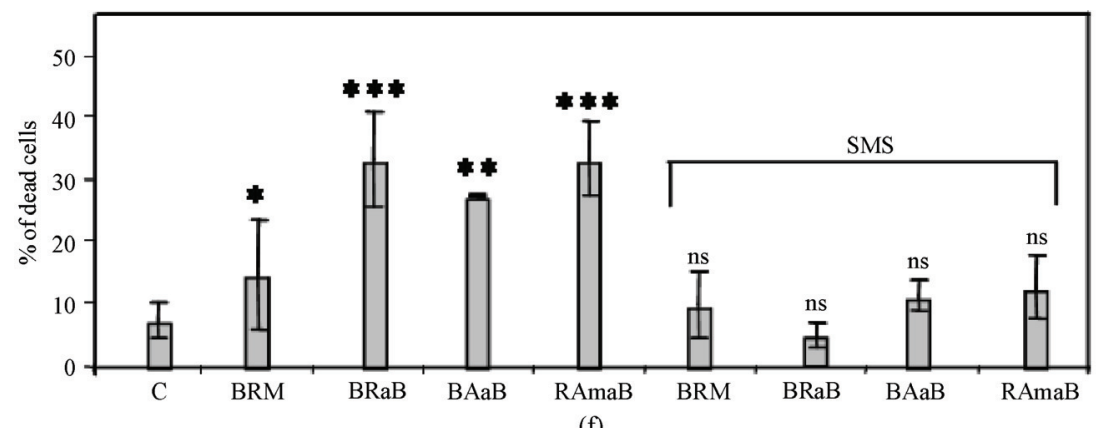

Figure 3. Effect of the 5-fold diluted BRM (panel a), 5-fold diluted BRaB (panel b), BAaB (panel c) and RAmaB (panel d) on the germination Index (GI\%) and on the cell viability (panel e and f) before and after (+SMS) the discolouring process. Lc: Lactuca sativa; Cs: Cucumis sativus; Sb: Sorghum bicolour. Statistical significance of germination index of seeds treated vs. seeds with no SMS treatment: ***p $<\mathbf{0 . 0 0 0 1}$. Panel e, indicates the number of adhering cells, panel $\mathrm{f}$, the percentage of dead cells evaluated by the trypan blue staining technique. Statistical significance of cell viability vs. cells with no chromo-bath addition (C: control): $* * * \mathrm{p}<0.0001 ; * * \mathrm{p}<0.001 ; * \mathrm{p}<0.05$; ns, not significant 
interesting trait of the here investigated enzyme was related to the capacity to catalyse discoloration at high ionic strength. Also this characteristic results to be of interest because of the nature of textile wastewaters that are often associated to high ionic strengths. In fact, the laccase from SMS discoloured acid chromo-baths in incubation media containing two different salts, $\mathrm{NaCl} 150$ $\mathrm{mM}$ and $\mathrm{NH}_{4} \mathrm{SO}_{4} 227 \mathrm{mM}$. The enzyme resulted to be $50 \%$ inhibited by $\mathrm{NaCl} 200 \mathrm{mM}$, but the discoloration of acid baths occurred in only $24 \mathrm{hrs}$. The enzyme showed also a significant stability with respect to the temperature of incubation, and all the characteristics previously described make the laccase from $P$. ostreatus SMS extremely attractive for industrial application, suggesting the exploitation of the SMS not only as a low cost substrate with oxidative capacity, but also as a source of robust laccase. In this context, the induction of laccase production could be advantageous for enzyme-purifying purpose, even though, in the experimental condition here explored, an effect of induction of the chromo-baths on the ligninolytic battery of enzymes eventually associated to the SMS was absent. Actually dyes induction of oxidative enzyme, comprising laccase, has been observed in fungi [31]. However, the process can not be defined a generic effect of dyes on fungal metabolisms [32]. On the other hand, results obtained comparing the isoforms of laccases from different supernatants by native electrophoresis showed that $P$. ostreatus was able to produce the enzyme (with similar profile) in very different growth conditions, spanning from the one related to the presence of an easily available carbon source (rich broth where the fungus grows in axenic culture), to growth condition with a more complex carbon source, eventually not sustaining the production of fruiting body and in presence of chromo-baths (onto SMS). Laccases from basidiomycetes have been described as capable to quite efficiently decolorize azo-dyes and, in relation to anthraquinonic dyes, the enzymes are reported as more efficient than other oxidases [33]. However, as far as we know, this is the first report on putative laccase capacity to discolour azo and anthraquinonic dyes in complex mixtures of chemicals as chromo-baths, containing high concentrations of different surfactants, anti-foams and salt. In relation to the experimental condition adopted, the extremely versatile laccase oxidative capacity here observed, can be interpreted as related to the co-presence of the enzyme and eventually of chemical redox mediators deriving from the ligninolytic activity of the basidiomycetes. Mediators act as electron transfer between the enzyme and very different substrates characterised by high redox state $[34,35]$. They can also accelerate oxidative processes in terms of kinetics of reactions involved [36,37]. Actually, intermediates of the process of lignin degradation, defined as natural mediators, are reported as the best one to use for dye discoloration by laccase [38]. With reference to the here described discolouring incubations, it is rea- sonable to assume that the ligninolytic activity of $P$. ostreatus, growing on a lignin containing substrate as the SMS, released these or analogous intermediates in the discolouring supernatants. Thus, the occurrence of natural mediators might be the reason of the peculiar capacity of the $P$. ostreatus SMS to discolour a plethora of different chromo-baths, with different chemical compositions, by the quite efficient kinetics of reactions here observed. Moreover, as a matter of fact, the Annex I of the Dangerous Substances Directive in the European Union (EU) describe some dye as toxic or mutagenic and others as skin sensitizers for consumers [Council Directive 67/ 548/EEC on the approximation of laws, regulations, and administrative provisions relating to the classification, packaging, and labeling of dangerous substances. Official Journal of the European Communities, June 27, 1967; Vol. 196, p 1.]. At the same time, some discolouring process are associated to the production of toxic intermediates of dyes oxidation process $[4,6]$. Thus, any approach for the treatment of textile wastewaters must be focused either on discolouring and detoxifying processes. Accordingly, the opportunity to exploit the SMS from $P$. ostreatus in the treatment of textile wastewaters has been verified by the toxicological assessment of the process, based on a cyto-toxicity test on a human amnion epithelial cell line (WISH) and a phyto-toxicity test on germinating seeds of Lactuca sativa, Cucumis sativus and Sorghum bicolor. The results obtained showed a strong toxicity of the chromo-baths related either to the cyto-toxicity exerted on cultured human amnion epithelial cells (WISH) and to the phyto-toxicity exerted on seed of different plant species. The discoloration of chromo-baths by the SMS was associated to the loss of toxicity either on WISH cells and plant seeds.

In conclusion, the SMS from $P$. ostreatus resulted to be capable to either discolour and detoxify a plethora of different chromo-baths containing complex mixtures of chemicals with recognised toxic effect for the environment. The versatility of the low cost organic substrate is related at least to the laccase activity deriving from $P$. ostreatus, even though other elements favouring oxidation cannot be excluded. All in all, the development of processes of treatment of textile wastewaters based on the exploitation of the substrate, either as a source of robust enzymes or as a versatile low cost organic substrate with oxidative capacity, results to be sustainable in terms of costs and eventually profitable for the design of an integrated management of the disposal of the SMS as an organic waste.

\section{Acknowledgements}

This work was supported by grants from Assessorato all'Istruzione, Formazione e Lavoro, Regione Toscana (Project MECHOS, POR Ob3 2000/2006 Toscana, Progetti integrati di ricerca Mis. D4) and from MIUR 
(Progetti di Rilevante Interesse Nazionale, PRIN 2007 to $\mathrm{RB})$.

\section{REFERENCES}

[1] T. Deveci, A. Unyayar and M. A. Mazmanci, "Production of Remazol Brilliant Blue R Decolourising Oxygenase from the Culture Filtrate of Funalia Trogii ATCC 200800," Journal of Molecular Catalysis B: Enzymatic, Vol. 30, No. 1, 2004, pp. 25-32.

[2] K. P. Sharma, S. Sharma, S. Sharma, P. K. Singh, S. Kumar, R. Grover and P. K. Sharma, "A Comparative Study on Characterization of Textile Wastewaters (Untreated and Treated) Toxicity by Chemical and Biological Tests," Chemosphere, Vol. 69, No. 1, 2007, pp. 48-54.

[3] I. M. Banat, P. Nigam, D. Singh and R. Marchant, "Microbial Decolourization of Textile Dyes Containing Effluents: A Review," Bioresource Technology, Vol. 58, No. 3, 1996, pp. 217-227.

[4] A. Stolz, "Basic and Applied Aspects in the Microbial Degradation of Azo Dyes," Applied Microbiology and Biotechnology, Vol. 56, No. 1-2, 2001, pp. 69-80.

[5] D. Brown and B. Hamburger, "The Degradation of Dyestuffs: Part III-Investigation of their Ultimate Degradability," Chemosphere, Vol. 16, No. 7, 1987, pp. 15391553.

[6] A. Gottlieb, C. Shaw, A. Smith, A. Wheatley and S. Forsythe, "The Toxicity of Textile Reactive Azo Dyes after Hydrolysis and Decolourisation," Journal of Biotechnology, Vol. 101, No. 1, 2003, pp. 49-56.

[7] J. A. Libra, M. Borchert, L. Vigelahn and T. Storm, "Two Stage Biological Treatment of a Diazo Reactive Textile Dye and the Fate of the Dye Metabolites," Chemosphere, Vol. 56, No. 2, 2004, pp. 167-180.

[8] J. S. Knapp, P. S. Newby and L. P. Reece, "Decolorization of Dyes by Wood-Rotting Basidiomycete Fungi," Enzyme and Microbial Technology, Vol. 17, No. 7, 1995, pp. 664-668.

[9] D. Wesenberg, I. Kyriakides and S. N. Agathos, "WhiteRot Fungi and their Enzymes for the Treatment of Industrial Dye Effluents," Biotechnology Advances, Vol. 22, No. 1-2, 2003, pp. 161-87.

[10] E. P. Chagas and L. R. Durrant, "Decolorization of Azo Dyes by Phanerochaete Chrysosporium and Pleurotus Sajorcaju," Enzyme and Microbial Technology, Vol. 29, No. 8, 2001, pp. 473-477.

[11] M. A. Martins, N. Lima, A. J. Silvestre and M. J. Queiroz, "Comparative Studies of Fungal Degradation of Single or Mixed Bioaccessible Reactive Azo Dyes," Chemosphere, Vol. 52, No. 6, 2003, pp. 967-973.

[12] J. Swamy and J. A. Ramsay, "The Evaluation of White Rot Fungi in the Decoloration of Textile Dyes," Enzyme and Microbial Technology, Vol. 24, No. 3-4, 1999, pp. 130-137.

[13] A. Heinfling, M. Bergbauer and U. Szewzyk, "Biodegradation of Azo and Phthalocyanine Dyes by Trametes Ver- sicolor and Bjerkandera adusta," Applied Microbiology and Biotechnology, Vol. 48, No. 2, 1997, pp. 261-266.

[14] G. Aggelis, D. Iconomou, M. Christou, D. Bokas, S. Kotzailias, G. Christou, V. Tsagou and S. Papanikolaou, "Phenolic Removal in a Model Olive Oil Mill Wastewater Using Pleurotus Ostreatus in Bioreactor Cultures and Biological Evaluation of the Process," Water Research, Vol. 37, No. 16, 2003, pp. 3897-3904.

[15] S. Rodríguez-Pérez, N. G. Oduardo, R. C. B. Savón, M. F. Boizán and C. Augur, "Decolourisation of Mushroom Farm Wastewater by Pleurotus Ostreatus," Biodegradation, Vol. 19, No. 4, 2008, pp. 519-526.

[16] L. H. Zhao, J. T. Zhou, H. Lu, C. L. Zheng, Y. S. Yang, H. J. Sun and X. H. Zhang, "Decolorization of Cotton Pulp Black Liquor by Pleurotus Ostreatus in a BubbleColumn Reactor," Bulletin of Environmental Contamination and Toxicology, Vol. 80, No. 1, 2008, pp. 44-48.

[17] V. Faraco, C. Pezzella, A. Miele, P. Giardina and G. Sannia, "Bio-Remediation of Colored Industrial Wastewaters by the White-Rot Fungi Phanerochaete Chrysosporium and Pleurotus Ostreatus and their Enzymes," Biodegradation, Vol. 20, No. 2, 2009, pp. 209-220.

[18] A. S. Ball and A. M. Jackson, "The Recovery of Lignocellulose Degrading Enzymes from Spent Mushroom Compost," Bioresource Technology, Vol. 54, No. 3, 1995, pp. 311-314.

[19] S. W. Chiu, S. C. Law, M. L. Ching, K. W. Cheung and M. J. Chen, "Themes for Mushroom Exploitation in the 21st Century: Sustainability, Waste Management, and Conservation," Journal of General and Applied Microbiology, Vol. 46, No. 6, 2000, pp. 269-282.

[20] M. R. Trejo Hernandez, A. L. Munguia and R. Q. Ramirez, "Residual Compost of Agaricus Bisporus as a Source of Crude Laccase for Enzymic Oxidation of Phenolic Compounds," Process Biochemistry, Vol. 36, No. 7, 2001, pp. 635-639.

[21] M. D. Karla, T. H. Maria del Refugio and R. P. Marco, "Recovery of Laccase from the Residual Compost of Agaricus Bisporus in Aqueous Two-Phase Systems," Process Biochemistry, Vol. 44, No. 4, 2009, pp. 435-439.

[22] H. G. Ko, S. H. Park, S. H. Kim, H. G. Park and W. M. Park, "Detection and Recovery of Hydrolytic Enzymes from Spent Compost of Four Mushroom Species," Folia Microbiologica, Vol. 50, No. 2, 2005, pp. 103-106.

[23] W. M. Law, W. N. Lau, K. L. Lo, L. M. Wai and S. W. Chiu, "Removal of Biocide Pentachlorophenol in Water System by the Spent Mushroom Compost of Pleurotus Puulmonarius," Chemosphere, Vol. 52, No. 9, 2003, pp. 1531-1537.

[24] M. M. Bradford, "A Rapid and Sensitive Method for the Quantitation of Microgram Quantities of Protein Utilizing the Principle of Protein-Dye Binding," Analytical Biochemistry, Vol. 72, 1976, pp. 248-254.

[25] A. M. Osman, K. K. Y. Wong, S. J. Hill and A. Fernyhough, "Isolation and the Characterization of the Degradation Products of the Mediator ABTS-Derived Radicals Formed upon Reaction with Polyphenols," Biochemical 
and Biophysical Research Communications, Vol. 340, No. 2, 2005, pp. 597-603.

[26] C. G. Boer, L. Obici, C. Marques de Souza and R. M. Peralta, "Purification and Some Properties of Mn Peroxidase from Lentinula Edodes Process," Process Biochemistry, Vol. 41, No. 5, 2006, pp. 1203-1207.

[27] M. Tien and T. K. Kirk, "Lignin-Degrading Enzyme from Phanerochaete Chrysosporium: Purification, Characterization, and Catalytic Properties of a Unique $\left.\left.\mathrm{H}_{2}\right) \mathrm{O}_{2}\right)$ Requiring Oxygenase," Proceedings of the National Academy of Sciences, Vol. 81, No. 8, 1984, pp. 22802284.

[28] F. Balestri, M. Giannecchini, F. Sgarrella, M. C. Carta, M. G. Tozzi and M. Camici, "Purine and Pyrimidine Nucleosides Preserve Human Astrocytoma Cell Adenylate Energy Charge under Ischemic Conditions," Neurochemistry International, Vol. 50, No. 3, 2007, pp. 517-523.

[29] Y. Fu and T. Viraraghavan, "Fungal Decolorization of Dye Wastewaters: A Review," Bioresource Technology, Vol. 79, No. 3, 2001, pp. 251-262.

[30] C. I. Pearce, J. R. Lloyd and J. T. Gurthrie, "The Removal of Colour from Textile Wastewater Using Whole Bacterial Cells: A Review," Dyes and Pigments, Vol. 58, No. 3, 2003, pp. 179-196.

[31] D. T. D'Souza, R. Tiwari, A. K. Sah and C. Raghukumar, "Enhanced Production of Laccase by a Marine Fungus During Treatment of Colored Effluents and Synthetic Dyes," Enzyme and Microbial Technology, Vol. 38, No. 3-4, 2006, pp. 504-511.

[32] S. Vanhulle, E. Enaud, M. Trovaslet, N. Nouaimeh, C. M. Bols, T. Keshavarz, T. Tron, G. Sannia and A. M. Corbisier, "Overlap of Laccases/Cellobiose Dehydrogenase
Activities during the Decolourisation of Anthraquinonic Dyes with Close Chemical Structures by Pycnoporus Strains," Enzyme and Microbial Technology, Vol. 40, No. 7, 2007, pp. 1723-1731.

[33] P. P. Champagne and J. A. Ramsay, "Contribution of Manganese Peroxidase and Laccase to Dye Decoloration by Trametes Versicolor," Applied Microbiology and Biotechnology, Vol. 69, No. 3, 2005, pp. 276-285.

[34] H. Claus, G. Faber and F. Koneig, "Redox-Mediated Decolorization of Synthetic Dyes by Fungal Laccases," Applied Microbiology and Biotechnology, Vol. 59, No. 6, 2002, pp. 672-678.

[35] P. Giardina, V. Farmaco, C. Pezzella, A. Piscitelli, S. Vanhulle and G. Sannia, "Laccases: A Never-Ending Story," Cellular and Molecular Life Sciences, Vol. 67, No. 3, 2010, pp. 369-385.

[36] B. Brogioni, D. Biglino, A. Sinicropi, E. J. Reijerse, P. Giardina, G. Sannia, W. Lubitz, R. Basosi and R. Pogni, "Characterization of Radical Intermediates in LaccaseMediator Systems. A Multifrequency EPR, ENDOR and DFT/PCM Investigation," Physical Chemistry Chemical Physics, Vol. 10, 2008, pp. 7284-7292.

[37] E. Dubé, F. Shareck, Y. Hurtubise, M. Beauregard and C. Daneault, "Decolourization of Recalcitrant Dyes with a Laccase from Steptomyces Coelicolor under Alkaline Conditions," Journal of Industrial Microbiology \& Biotechnology, Vol. 35, No. 10, 2008, pp. 1123-1129.

[38] S. Camarero, D. Ibarra, M. J. Martinez and A. T. Martinez, "Lignin-Derived Compounds as Efficient Laccase Mediators for Decolorization of Different Types of Recalcitrant Dyes," Applied and Environmental Microbiology, Vol. 71, No. 4, 2005, pp. 1775-1784.

\author{
Abbreviations: \\ SMS, Spent Mushroom Substrate; \\ BRM, Black chromo-Reactive Mix; \\ $\mathrm{BRaB}$, Blue chromo-Reactive anthraquinonic Bath; \\ $\mathrm{BAaB}$, Blue chromo-Acid anthraquinonic Bath; \\ RAmaB, Red chromo-Acid monoazo Bath; \\ ABTS, 2,2'-azinobis-(3-ethylbenzthiazoline-6-sulfonic) acid.
}

